\title{
RHINOSCLEROMA WITH LARYNGOTRACHEAL INVOLVEMENT, CAUSING DIAGNOSTIC DILEMMA
}

Chandrakant Patil, Rashmi Patil (Kharat), Jyotirmoy Biswas, Vijayshree Nahata

\author{
1. Professor, Department of ENT, Jawaharlal Nehru Medical College, DMIMSU, Warda. \\ 2. Associate Professor, Department of ENT, Jawaharlal Nehru Medical College, DMIMSU, Warda. \\ 3. Resident, Department of ENT, Jawaharlal Nehru Medical College, DMIMSU, Warda. \\ 4. Resident, Department of ENT, Jawaharlal Nehru Medical College, DMIMSU, Warda.
}

\section{CORRESPONDING AUTHOR}

Dr. Chandrakant Patil

Professor, Dept. of ENT,

JNMC, DMIMSU, Sawangi (m), Warda-442002

E-mail: dr_chandupatil@rediffmail.com

Ph: 00919370851476

ABSTRACT: Rhinoscleroma is a chronic, progressive, granulomatous disease caused by Klebsiella Rhinoscleromatis. It generally involves the nose but can affect other parts of the respiratory tract. It is uncommon but still evident in some parts of the world. We are reporting a case of a 25 year-old male who presented with nasal mass, dyspnoea and hoarseness of voice and created a diagnostic dilemma due to laryngeal involvement but histopathological examination revealed the diagnosis of rhinoscleroma. Apart from surgical debridement of the mass in nasal cavity, the patient was also treated with streptomycin, rifampicin and ciprofloxacin. He was asymptomatic in the follow-up. It is thus important to consider rhinoscleroma in cases of chronic nasal obstruction and histopathology plays a vital role in confirming the diagnosis.

KEY WORDS: rhinoscleroma, laryngotracheal scleroma, change in voice.

INTRODUCTION: Rhinoscleroma is a progressive granulomatous disease commencing, mainly in nose and later extending into the nasopharynx, the oropharynx, the larynx \& sometimes the trachea or even the bronchi. Laryngeal involvement can be seen in almost half of the cases. [1] Laryngeal and tracheal involvement requires special attention as they carry high risk. The dysphonia, dyspnoea, strider and cough suggest laryngotracheal involvement.[2]

This disease caused by gram negative bacillus, Klebsiella rhinoscleromatis, a subspecies of Klebsiella pneumoniae. Commonly seen in central \& south-eastern Europe, North Africa, America, Indian subcontinent, South-East Asia including Indonesia \& China [2], [3] and human beings are the only known host.

Here, we present one such case with chronic nasal obstruction and change of voice, indicating both nasal and laryngeal involvement, without any prior particular diagnosis.

This prompted us to report our experience on the clinicopathological aspect of this disease.

CASE REPORT: A 26 years old male farmer presented with complains of bilateral nasal obstruction since 6 years \& change of voice since 1 year. Nasal blockade was insidious, progressive, and bilateral more on left side, associated with nasal discharge and crusting. Change in voice was non-progressive, not associated with throat pain, fever, difficulty in swallowing. Patient was treated by a general practitioner for recurrent URTI but with no relief. 
On local examination a granular mass was seen in both the nasal cavities more on the left side. (fig.1) On indirect laryngoscopy examination there was a submucosal mass or thickening seen in the region of false cord on right side.

Routine blood investigations were in normal range except ESR was raised (85mm/hr).X ray chest was also normal. C.T. scan of PNS and larynx revealed mass in both nasal cavity without involvement of PNS (fig.2),and a mass in the right Supraglottic region with some concentric narrowing of subglottic and upper part of the trachea.(fig.3).

With the possibility of malignancy in mind, under General anaesthesia excisional biopsy of the nasal mass was done with the help of a microdebrider. Direct laryngoscopy was also performed and biopsy taken from supraglottic \& subglottic region. Direct laryngoscopy also revealed oedema in the Upper part of trachea.

Histopathology examination of biopsies from both sides revealed chronic inflammatory reaction, foamy histiocytes admixed with inflammatory cells mainly plasma cells, and Russell bodies. (Fig-4), which confirmed the diagnosis of rhinoscleroma.

Patient was treated initially with Inj. Streptomycin (1 gm OD for 1 week) and Tab. Rifampicin (600 mg OD for 6 weeks), Subsequently, Tab. Ciprofloxacin was started for 4 months. With regular follow up, patient showed improvement and was symptom free.

DISCUSSION: The term "Rhinoscleroma" was first coined in 1870 by Von Hebra, who described a nasal lesion that was classified as a form of sarcoma. In 1877, Mikulicz described the histological features of this disease in detail and established its non-neoplastic, inflammatory nature. Von Frisch identified the causal agent of this lesion in 1882 as a gram-negative coccobacillus. ${ }^{[3]}$

It is important to consider rhinoscleroma a differential diagnosis in cases of chronic nasal obstruction. [4] In case of Rhinoscleroma, Poor hygiene, crowded living conditions, malnutrition and immunocompromised states are predisposing factors [5], however the patient in present study was immunocompetent. Rhinoscleroma is a chronic progressive granulomatous disease mostly affecting nose. In a clinicopathological study done in gulf region 6 out of 25 patients were found to have nasopharyngeal involvement whereas 11 cases showed laryngeal involvement. ${ }^{[2]}$ Some authors showed Tracheal involvement in only $12 \%$ of cases.[6]

Typically, rhinoscleroma progresses through three clinical stages. Initially a catarrhal phase is characterised by purulent and fetid rhinorrhea, which may progress to nasal mucosal atrophy and crusting. The second phase is a granulomatous phase in which nodules or masses may lead to obstructive symptoms in the respiratory tract. The final stage is a cicatricial stage of scarring which may cause external deformity or stenosis of nasal passage or laryngeal airway. ${ }^{[7]}$ The differential diagnosis of respiratory scleroma includes nasal tumours and other tumours of respiratory tract, fungal infections, specific granulomatous diseases like tuberculosis and leprosy. Wegener's granulomatosis, T cell lymphomas, sarcoidosis and Basal cell carcinomas may have similar presentations [7].

The symptoms are nonspecific such as mucopurulent rhinorrhea, cough, breathing difficulty due to nasal obstruction, dysphonia, epistaxis and headache. [4] Our patient also presented similarly and was treated non specifically by general practitioners creating a delay in the diagnosis hence treatment.

In literature review of respiratory tract scleroma, a case of scleroma presenting as an upper tracheal mass causing stridor and respiratory distress was described, The patient was managed with urgent resection and an eight week course of oral ciprofloxacin hydrochloride . 
The author commented that there were only two cases of isolated laryngotracheal scleroma, both of which required urgent tracheostomy. ${ }^{[8]}$

In Los Angeles study of 22 patients with scleroma [9], 59\% of patients had laryngotracheal scleroma. All patients in this study had nasal mucosal involvement. Patients presented with dysphonia, exertional dysnoea and or stridor. Patients in this series were initially managed with intravenous antibiotics and dexamethasone and three required tracheostomies. All patients were in the granulomatous phase of infection, and it appeared that granuloma formation was more common in the glottis than in the subglottis. A case of a severe form of laryngotracheal rhinoscleroma, which resulted in the death of the patient, is also described in literature [10]. A case of subglottic laryngeal stenosis has also been described as a late sequel of the disease after 15 years of diagnosis of rhinoscleroma. [11]

Treatment should include a long-term antimicrobial therapy and surgical intervention in cases with symptomatic obstruction. [5] Drugs of choice include quinolones, doxycycline or tetracycline and less frequently streptomycin and rifampicin.[12-14] Rifampicin provides good results in the treatment of rhinoscleroma, but requires effective monitoring of toxicity.[15] Because of high rate of relapses the long-term follow-up of the patient is necessary. Being aware of clinical presentations, early diagnosis of this condition is the prerequisite to reduce the morbidity caused by this disease. [5]

The correct diagnosis is given based on histopathological findings and the clinical history, which includes information on living conditions and the work environment. [14]

In this case patient's nasal obstruction was treated with debridement of nasal mass and chemotherapeutic agents. Laryngeal symptoms were not addressed surgically as being rural tertiary centre, availability of laryngeal stent was one and as patient was not having stridor was another issue in favour of long term medical treatment to prevent relapse.

The objective of presenting this case is to highlight a disease process that is generally identified predominantly as a cause of nasal disease, can also involve the larynx and trachea and may present with symptoms of involvement of these organs or part of body. This can create a diagnostic dilemma.

CONCLUSION: A high index of suspicion and histopathological examination is of paramount importance to diagnose a case of nasal mass with laryngeal involvement to prevent diagnostic dilemma.

\section{REFERENCES:}

1. S Bahadur, A Thakar.In:Valerie J Lund Editor.Scott-Brown's Otorhinolaryngology, Head and Neck Surgery, $7^{\text {th }}$ ed.Vol.2 UK: Edward Arnold pub.ltd; 2008. p 1462-1463

2. Loic de Pontual, Philippe O, Diana R, Audrey G, Anne P, Jacinta B, Sabine P, Laurent Y, Pierre-Yves L, Daniele D, Michel Huerre, Regis T, Philippe S, Laurent A, Jean LaurentCasanova; Rhinoscleroma: A French National Retrospective Study of Epidemiological and Clinical Features; Clinical infectious diseases. 2008;47: 1396-1402

3. Abalkhail A, Satti M B, Uthman M A E, Al Hilli F, Darwish A, Satir A; Rhinoscleroma: a clinicopathological study from the Gulf region; Singapore Med J 2007; 48(2): 148-151

4. Mario A P de M, Albino V de M, Larissa C M, Ana E B de A, Fabiana P C, Igor T R; Rhinoscleroma causing severe bilateral nasal obstruction; Braz J Infect Dis 2010; 14(2): 190-192 
5. Deepak A, M Maqbool, Fir Afroz, M Ashraf, N A Khan; Rhinoscleroma Mimicking Malignancy; J K Science, 2008:10(1):32-33.

6. Arunabh T, N Patel, Lisa C, Rakesh S, Donald M; Rhinoscleroma of the Tracheobronchial Tree: Bronchoscopic, PET, and CT Correlation;The Indian Journal of Chest Diseases \& Allied sciences. 2008;50 (2): 225-228.

7. G Verma, D Kanawaty, R Hyland.Rhinoscleroma causing upper airway obstruction. Can Respir J 2005;12(1):43-45.

8. Alfaro -Monge JM, Fernandez-Espinosa J, Soda-Merhy A. Scleroma of the lower respiratory tract: Case report and review of literature. J Laryngol Otol 1994; 108:161-3.

9. Amolis CP, Shindo ML. Laryngotracheal manifestations of rhinoscleroma. Ann Otol Rhinol Laryngol 1996; 105:336-40.

10. Yigla M, Ben-Izhak O, Oren I, Hashman N, Lejbkowicz F. Laryngotracheaobronchial involvement in a patient with nonendemic rhinoscleroma. Chest 2000 ;117:1795-98.

11. D. Munoz-Saavedra,C.Olavarria-leiva Laryngeal stenosis as late manifestation of rhinoscleroma. Acta Otorrhinolaringol Esp.2010;61(3):241-243.

12. BadiaL,Lund VJ.Acase of rhinoscleroma treated with ciprofloxacin.J Laryngol Otol 2001;115:220-2.

13. Bhargava D,Date A.Palatal presentation of scleroma. J Laryngol Otol 2001;115:679-80.

14. Ammar ME,Rosen A. Rhinoscleroma mimicking nasal polyposis.Ann otol Rhinol Laryngol 2001;110:290-2.

15. Monica ES, Lidio G, Roberto CBO, Monica PAA; Rhinoscleroma: Case Report; Brazilian Journal of Othorhinolaryngology2006; 72 (4) : 568-71.

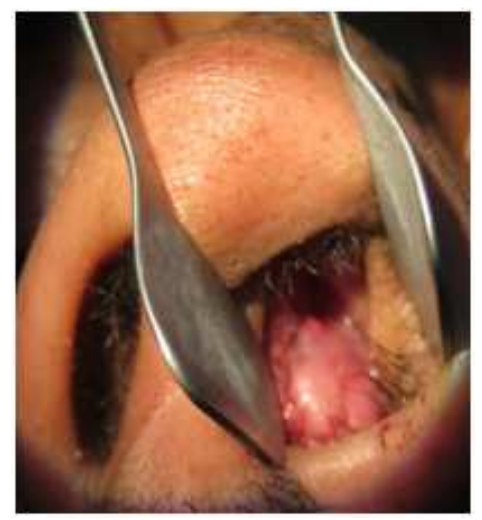

fig.1 Granular mass in Lt. nasal cavity

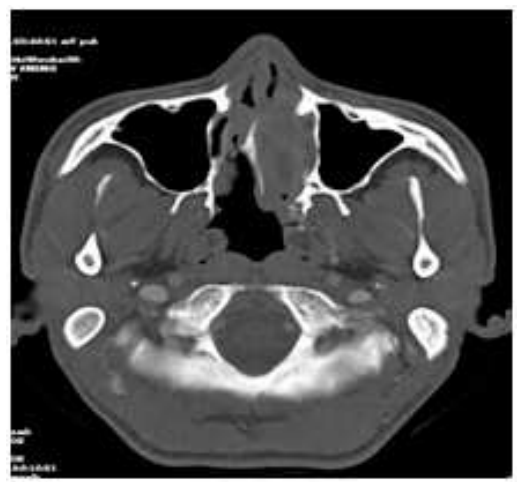

Fig.2 C.T.PNS showing mass in both nasal cavity more on Lt. side without involvement of PNS. 

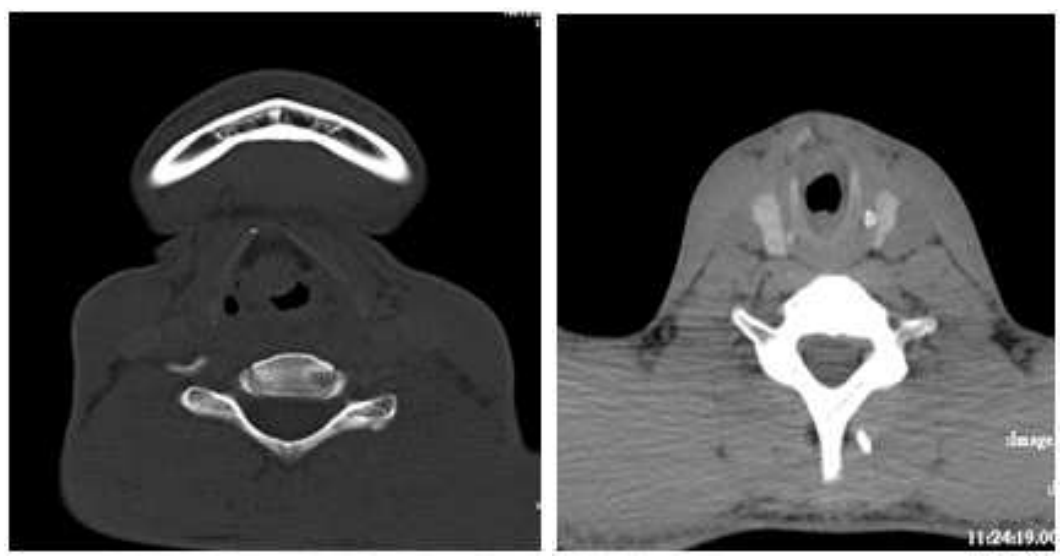

Fig.3. C.T. Larynx showing submucosal mass in Rt. supraglottic region, also concentric narrowing of subglottis and upper part of trachea.

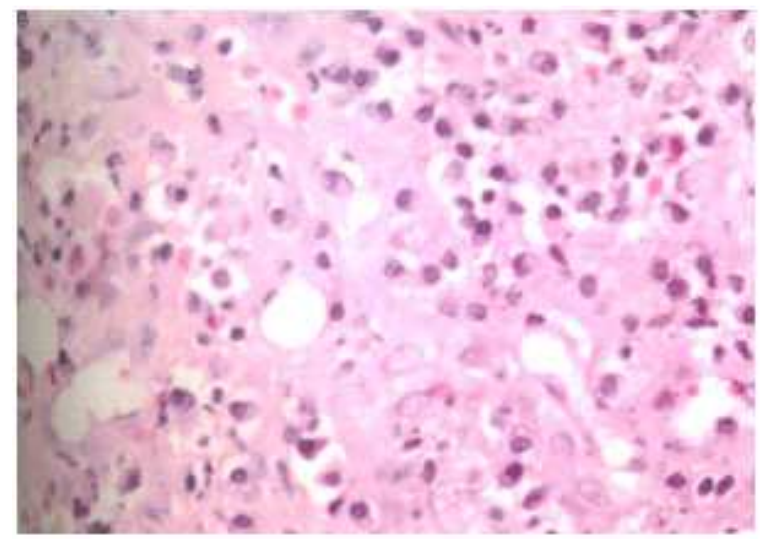

Fig.4. Histopathology s/o chronic inflammation with large vacuolated histiocytes, (foamy histiocytes) plasma cells and Russell bodies.(H\&E 200 x) 